Philosophie ANTIQUE

\section{Philosophie antique}

Problèmes, Renaissances, Usages

$18 \mid 2018$

L'athéisme antique

\title{
Angela LONGO et Daniela Patrizia TAORMINA (éd.), Plotinus and Epicurus. Matter, Perception, Pleasure
}

\section{Alexandra Michalewski}

\section{OpenEdition}

\section{Journals}

Édition électronique

URL : https://journals.openedition.org/philosant/1127

DOI : 10.4000/philosant. 1127

ISSN : 2648-2789

\section{Éditeur}

Éditions Vrin

\section{Édition imprimée}

Date de publication : 1 novembre 2018

Pagination : 297-300

ISBN : 978-2-7574-2372-1

ISSN : $1634-4561$

Référence électronique

Alexandra Michalewski, « Angela Longo et Daniela Patrizia taormina (éd.), Plotinus and Epicurus. Matter, Perception, Pleasure », Philosophie antique [En ligne], 18 | 2018, mis en ligne le 01 novembre 2018, consulté le 03 décembre 2022. URL : http://journals.openedition.org/philosant/1127 ; DOI : https:// doi.org/10.4000/philosant. 1127

\section{(c) (i) (3)}

Creative Commons - Attribution - Pas d'Utilisation Commerciale - Pas de Modification 4.0 International - CC BY-NC-ND 4.0

https://creativecommons.org/licenses/by-nc-nd/4.0/ 
originelle du principe. L'analyse que propose l'auteur de l'efficacité de ce modèle pour penser l'existence de l'Un est très originale et convaincante. Elle doit toutefois être complétée par le rappel d'un scrupule ultime qui saisit Plotin dans les derniers chapitres du traité : le modèle de la coexistence se trouve désormais délaissé, puisque l'Un apparaît alors « seul, vraiment libre (...) alors que chacun des autres êtres est à la fois lui-même et autre chose » (21,31-33). Au modèle de la coexistence il faut désormais préférer la position d'une pure solitude qui coïncide avec la liberté première. La métaphysique de l'Un ne saurait s'en tenir à subvertir le paradigme substratif de l'ontologie d'Aristote : elle instaure un nouveau régime de la métaphysique qui rompt radicalement avec tout modèle d'articulation interne de l'être, qu'il s'agisse du substrat, de l'inclusion réciproque ou de la coexistence.

LAURENT LAVAUD

Université de Paris 1-PAnthéon Sorbonne/UMR 7219

\section{A. Longo \& D. P. Taormina (éd.), Plotinus and Epicurus. Matter, Perception,} Pleasure, Cambridge, Cambridge University Press, 2016, 236 p., ISBN : 978-1-10712421-9.

Des grands courants philosophiques de l'époque hellénistique que Plotin reprend et discute, l'épicurisme est, pour ainsi dire, le parent pauvre. Aussi pendant longtemps, la littérature secondaire avait-elle pratiquement négligé l'étude de sa réception dans les Ennéades. En s'emparant de cette question, cet ouvrage collectif fait bien davantage que pallier une lacune scolastique. Il s'inscrit dans une perspective ouverte récemment visant à mettre en lumière les points sur lesquels l'épistémologie plotinienne reprend et adapte certains éléments issus de l'épicurisme. Elle s'était développée il y a une dizaine d'années, à partir d'une discussion autour du statut de l'ennoia dans le traité 45 (III, 7) : inversant la lecture traditionnelle selon laquelle toute ennoia serait la trace dans l'âme des Formes transcendantes, B. van den Berg (« As we are always speaking of them and using their names on every occasion ... », dans R. Chiaradonna \& F. Trabattoni (éd.), Physics and Philosophy of Nature in Greek Neoplatonism, Leiden, 2009) soutenait que, selon Plotin, l'ennoia du temps aurait, à la différence de l'ennoia de l'éternité, une origine empirique. P. Remes (« Plotinus on Starting Points of Reasoning », Chôra 14, 2016) reprenant cette question, avait ensuite examiné plus spécialement la place que Plotin confere à certaines conceptions, formées à partir de l'expérience, qui, sans être des critères du vrai, ne sont pas non plus dépourvues de toute fiabilité, puisqu'elles peuvent servir de points de départ à la réflexion et à l'analyse philosophiques. Ce volume, consacré entièrement aux multiples aspects du rapport que Plotin entretient avec Épicure et l'épicurisme, approfondit considérablement les recherches dans cette voie. Mais ce n'est là qu'un aspect de la question. Plusieurs de ses contributions proposent de lire certains passages des Ennéades, tels que Enn. V, 5 (32), 1 ou Enn. III, 2 (47), 7, comme des éléments repérables du dossier anti-épicurien, révélant l'existence d'un véritable dialogue avec l'épicurisme dans les traités. Enfin et surtout, il permet d'aller bien au-delà des pistes ouvertes en 1981 par J.-P. Dumont (« Plotin et la doxographie épicurienne », dans Néoplatonisme, Mélanges offerts à Jean Trouillard, 1981) qui, s’intéressant aux allusions à l'atomisme dans les traités, restreignait la connaissance que Plotin avait de l'épicurisme aux simples compilations doxographiques.

Le recueil comporte, outre l'introduction, dix contributions, réparties en quatre sections de taille inégale. Entre la première, qui est un aperçu historique extrêmement bien documenté de T. Dorandi concernant l'état de la diffusion des écrits d'Épicure à 
travers les grandes villes de la Méditerranée jusqu’au début du IV ${ }^{\mathrm{e}}$ siècle - et la dernière, constituée par deux articles abordant les points de contact entre Plotin et certaines thèses épicuriennes -, les deux sections centrales regroupent des articles qui analysent les différents aspects et niveaux de la polémique anti-épicurienne. L'article inaugural de T. Dorandi brosse l'état de la situation et pose une hypothèse qui sera exploitée par nombre de contributions du volume. Prenant parti dans la délicate question de la succession institutionnelle du Jardin, il argumente en faveur de la perpétuation de diadoques dans l'Athènes $\mathrm{du} \mathrm{II}^{\mathrm{e}}$ siècle (p. 35); s'appuyant sur la récente publication $\mathrm{du}$ POxy. 5077, qui atteste que les lettres d'Épicure étaient lues et discutées jusqu'au III ${ }^{\mathrm{e}}$ siècle (p. 40-48), l'article souligne que l'épicurisme était encore très vivace à l'époque de Plotin dans une province cultivée comme l'Égypte, apportant de nouveaux éléments aux pistes tracées par F.-M. Smith dans les années 1990. L'hypothèse de T. Dorandi est qu'il est raisonnable de penser que Plotin avait non seulement accès aux résumés doxographiques mais aussi aux textes mêmes d'Épicure et qu'il avait pleine connaissance de ce courant de pensée qui comptait encore comme l'un des plus grands sur la scène philosophique de son temps.

La deuxième section (Common Anti-Epicurean Arguments in Plotinus) comporte à elle seule la moitié des contributions et donne à voir l'éventail des différents niveaux de polémique. Les trois premiers articles de cette section attirent l'attention sur la manière dont s'articulent, dans les traités, les diatribes anti-gnostiques aux critiques antiépicuriennes. Pour commencer, A. Longo montre comment, dans le traité II, 9 (33), la critique des thèses épicuriennes, qui associe de manière volontairement polémique le refus de la providence à la poursuite du plaisir, a un rôle instrumental, puisquelle sert essentiellement à renforcer la critique des thèses gnostiques. A. Longo poursuit en notant que cette fonction dévolue à Épicure - servir de support à la caricature de thèses d'opposants ciblés - est en réalité une sorte de lieu commun à l'époque, qu'on retrouve aussi chez les chrétiens d'Alexandrie, tel Origène qui, utilisant cette même connexion entre la négation de la providence et la poursuite du plaisir, voit en Épicure la quintessence de l'abomination des thèses païennes. Poursuivant sur la question de la providence, l'article de M. Mazzetti cherche à déterminer quel est l'adversaire visé par Plotin en Enn. III, 2 (47), 7. Dégageant l'arrière-plan argumentatif du chapitre, qui repose sur les arguments de Lois, $\mathrm{X}$ dirigés contre ceux qui, tout en admettant les dieux, refusent néanmoins l'existence de la providence, M. Mazzetti relie le chapitre 7 aux lignes d'ouverture du traité, directement dirigées contre les épicuriens. D’après lui, la cible de Plotin, ce ne sont pas les péripatéticiens, mais bien les épicuriens et les gnostiques qui considèrent que la providence est incapable de s'exercer sur terre. Si la thèse de M. Mazzetti est très convaincante, on regrette néanmoins qu'il n'aborde pas davantage la question du rapport de Plotin à Alexandre d'Aphrodise qui permettrait de mieux saisir tous les éléments du dossier, et de comprendre par exemple pourquoi sur cette question Plotin développe une approche si différente de celle d'Atticus. La contribution de M. P. Marsola, partant de la critique adressée par Plotin en Enn. V, 9 (5), 1 , à l'encontre de ceux qui consacrent leur vie à la seule recherche du plaisir, se propose de tracer un parallèle entre la critique anti-épicurienne et la critique anti-gnostique.

Après avoir traité des questions cosmologiques et éthiques, les contributions de P.-M. Morel et D. P. Taormina, centrées sur les questions épistémologiques, examinent toutes deux le premier chapitre d'Enn. V, 5 (32), où Plotin développe la critique de la thèse selon laquelle la connaissance dérive de la sensation. Tous deux, quoique à des degrés divers, reprennent la thèse que Bréhier avait exposée dans sa notice du traité 32, selon laquelle le premier adversaire de Plotin dans ce chapitre est l'épicurisme. Selon 
P.-M. Morel, dans ce chapitre, si Plotin a en vue les épicuriens, ou peut-être une certaine présentation de l'épicurisme servant à camper avec plus de force la polémique, c'est dans la mesure où ils représentent le mieux la thèse générale de l'empirisme. L'article offre une étude subtile qui apporte en effet plusieurs nuances dans les lectures respectives de Plotin et d'Épicure. Il rappelle tout à la fois que Plotin ne déconsidère pas la sensation en tant que telle, mais sa survalorisation épistémologique qui fait de l'évidence sensorielle un critère du vrai et qu'Épicure ne prône pas un simple sensualisme, mais plutôt un « empirisme rationnel »(p. 112) qui, fondé sur l'évidence, ne rejette pas les raisonnements en tant que tels, mais incite à la prudence à l'égard des mauvaises conclusions qui peuvent en découler. D. P. Taormina, pour sa part, reprenant le dossier concernant le statut de la perception et la nature des arguments sceptiques développés aux lignes 12-19, se propose d'apporter confirmation à l'ancienne hypothèse de Bréhier. Elle met particulièrement bien en lumière l'unité de la structure en deux temps de l'argument anti-épicurien de Plotin : elle montre bien comment l'argument selon lequel nous percevons seulement nos propres affections et non les qualités des objets (dont elle rappelle qu'il constitue un lieu commun des critiques platoniciennes des théories atomistes de la perception), ne constitue qu'un premier moment de l'objection et ne saurait être tenu pour la position dernière de Plotin concernant le caractère réaliste ou non de la perception ; ce sont les lignes suivantes qui portent le coup de grâce à la théorie épicurienne de la sensation : celle-ci reste toujours extérieure à son objet puisqu'elle ne donne accès qu'aux eidola, simples copies des réalités véritables.

La troisième section, qui comporte deux articles, est consacrée aux points fondamentaux d'antagonisme entre le platonisme de Plotin et l'atomisme d'Épicure (Plotinus' criticism of Epicurean doctrines), entre une pensée qui fait dépendre l'ensemble du réel d'un unique principe et un système dans lequel tout repose sur l'éclatement principiel des corps en mouvement perpétuel. La contribution de M. Ninci examine les deux aspects de la critique plotinienne de la matière atomiste. S'agissant de la perspective physique, M. Ninci montre très bien comment Plotin puise dans les analyses aristotéliciennes, notamment celles du continu, pour s'opposer à la conception épicurienne de la matière (p. 134-139). Du point de vue métaphysique (p. 142), Plotin souligne que la matière, qui ne possède aucune qualité, est incapable de produire par ellemême une réalité différente d'elle. Les corps apparaissent grâce à la forme qui est projetée sur la matière par une instance démiurgique. La récupération par Plotin de l'imagerie démiurgique dans ce contexte de polémique est particulièrement bien étudiée par M. Ninci. La question de l'origine de la détermination des corps est en effet directement liée à la question de l'existence ou non d'un principe intelligent. La contribution d'E. Eliasson est ainsi consacrée à la question de la providence. Centré sur l'analyse d'Enn. III, 1 (3), l'article examine les arguments exposés par Plotin visant à montrer que seule l'unité d'un principe premier permet d'échapper au déterminisme aveugle auquel condamne nécessairement la pluralité principielle.

La dernière section aborde la question des aspects de l'épicurisme que Plotin reprend pour les adapter à sa propre philosophie (Epicurean Elements in Plotinus) - ce qui constitue évidemment un domaine assez restreint. A. Cornea, qui traite de l'usage dans les traités plotiniens de la formule athroa epibole, issue de la Lettre à Hérodote, émet l'hypothèse d'une connaissance directe du texte épicurien par Plotin, et détecte, à travers cet exemple, l'indice d'une indéniable vivacité de l'épicurisme sur la scène philosophique de la fin du III e siècle. À la p. 184, il aborde la question discutée de la traduction de la

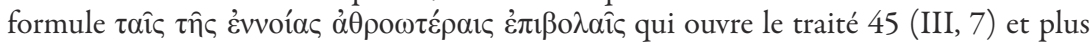
spécialement de l'adjectif athroos qui peut renvoyer à une saisie supra-discursive ou, à 
l'inverse, infra-discursive. K. Strange avait le premier attiré l'attention sur l'origine épicurienne de la formule, suivi ensuite par van den Berg. Cornea qui, semble-t-il, n'a pas connaissance de l'article de van den Berg, note pour sa part qu'athroos est un terme oxymorique qui indique une approche à la fois totale et immédiate. Il choisit de le traduire par « concentré » (《 concentrated »; «packed together »). La dernière contribution, d’A. Linguiti, est consacrée à la question du bonheur. Après avoir souligné les éléments d'opposition d'Épicure et de Plotin sur ce point - l'un tenant qu'il est identique au plaisir corporel, l'autre qu'il est identique à la contemplation des Formes - Linguiti rappelle que tous deux partagent néanmoins une même vision du rapport bonheur-temps : le bonheur, qui est immédiatement parfait, ne s'accroît pas avec le temps. Cette thèse, partagée aussi par les stoïciens, a toutefois chez Plotin une double fondation. L'une est métaphysique : le bonheur ne croît pas avec le temps puisqu'il coïncide avec l'activité de l'âme non descendue qui est extra-temporelle. La seconde, par où Plotin d'une certaine manière rejoint les épicuriens, consiste à dire que le bonheur éprouvé par le sujet humain dans le présent est parfait en soi-même et indépendant de la durée.

Pour conclure, ce volume, de grande qualité, permet de jeter un regard nouveau sur le rapport de Plotin à l'épicurisme dans la mesure non seulement où il donne des éléments permettant de soutenir l'hypothèse d'une connaissance directe d'Épicure par Plotin, mais aussi parce qu'il examine avec une grande précision les usages stratégiques et polémiques que Plotin en tire dans les Ennéades.

Alexandra Michalewski CNRS - CENTRE LÉON-Robin (UMR 8061)

Ilaria L. E. Ramelli, Social Justice and the Legitimacy of Slavery. The Role of Philosophical Asceticism from Ancient Judaism to Late Antiquity, Oxford, Oxford University Press, 2016 (Oxford Early Christian Studies), xvi + 293 p., ISBN : 978-019-87727-4.

Le présent volume vient s'ajouter à la bibliographie déjà très abondante d'Ilaria Ramelli ; elle y aborde une nouvelle fois ses auteurs de prédilection, en particulier Origène et les Cappadociens, qu'elle rassemble sous l'étiquette d'ascétisme philosophique chrétien. L'auteur reprend ici sa méthode habituelle, qui procède par amalgames et répétitions, afin de faire à la fin admettre sa thèse, sans pour autant l'avoir proprement démontrée. L'ouvrage aborde cette fois la question de l'esclavage, et plus particulièrement les positions en faveur de son abolition, dans la philosophie antique et le christianisme ancien. L'extension du domaine couvert paraît démesurée, au vu de la taille de l'ouvrage : philosophie grecque et hellénistique - et il faut y ajouter une partie de la philosophie d'époque impériale -, judaïsme biblique et rabbinique, christianisme ancien - curieusement distingué des « auteurs patristiques grecs, latins et syriaques ». La thèse centrale est la suivante : le rejet total de l'esclavage et le souhait vigoureux de son abolition seraient le fait d'un groupe déterminé de juifs et surtout de chrétiens de l'Antiquité, ascètes, et marqués par la philosophie («Christian ascetic-philosophers »). Le plan de l'ouvrage est le suivant : l'introduction, intitulée « La question débattue, orientations méthodologiques et apport à la recherche », présente de manière rhapsodique à la fois des éléments de définition, souvent énumératifs, le rappel de certains travaux antérieurs - dont l'auteur souligne plusieurs fois qu'ils s'accordent avec elle, alors qu'on attendrait plutôt la formulation inverse - mais aussi un développement sur les Sentences de Sextus, dont on comprend mal pourquoi il est placé ici. Le premier chapitre concerne « l'arrièreplan de la philosophie grecque et du judaïsme ancien »; le deuxième, « Le Nouveau 